\title{
A genome-wide association study of copy-number variation identifies putative loci associated with osteoarthritis in Koreans
}

\author{
Sanghoon Moon ${ }^{1 \dagger}$, Bhumsuk Keam ${ }^{1,2+}$, Mi Yeong Hwang ${ }^{1}$, Young Lee ${ }^{1}$, Suyeon Park ${ }^{1,4}$, Ji Hee Oh¹, \\ Yeon-Jung Kim', Heun-Sik Lee', Nam Hee Kim', Young Jin Kim', Dong-Hyun Kim³, Bok-Ghee Han', \\ Bong-Jo Kim ${ }^{1}$ and Juyoung Lee ${ }^{1 *}$
}

\begin{abstract}
Background: OA is a complex disease caused by environmental and genetic risk factors. The purpose of this study is to identify candidate copy number variations (CNVs) associated with OA.

Methods: We performed a genome-wide association study of CNV to identify potential loci that confer susceptibility to or protection from OA. CNV genotyping was conducted using NimbleGen HD2 $3 \times 720 \mathrm{~K}$ comparative hybridization array and included samples from 371 OA patients and 467 healthy controls. The putative CNV regions identified were confirmed with a TaqMan assay.

Results: We identified six genomic regions associated with OA encompassing CNV loci. None of six loci had previously been reported in genome-wide association studies with $\mathrm{OA}$, although a genetic analysis suggested that they have functional effects. The protein product of a candidate risk gene for obesity, TNKS, targets Wnt inhibition, and this gene was significantly associated with hand and knee OA. Copy number deletion on TNKS was associated with a 1.37-fold decreased risk for OA. In addition, CA10, which shows a strong association with osteoporosis, was also significant in our study. Copy number deletion on this gene was associated with a 1.69-fold decreased risk for OA.
\end{abstract}

Conclusion: We identified several CNV loci that may contribute to OA susceptibility in Koreans. Further functional investigations of these genes are warranted to fully characterize their putative association.

Keywords: Osteoarthritis, Copy number variation, OA risk gene, TNKS, CA10

\section{Background}

Osteoarthritis $(\mathrm{OA})$, which is also known as degenerative arthritis, is a disease of mechanical dysfunction involving degradation of joint components, especially articular cartilage and subchondral bone [1]. OA is the most common form of arthritis that affects elderly people and often leads to physical disability and reduced quality of life. OA is a leading cause of impaired mobility in the elderly [2]. The clinical course of OA varies from minimal joint pain, stiffness, and limitations in function to complete disability.

\footnotetext{
*Correspondence: jylee@cdc.go.kr

${ }^{\dagger}$ Equal contributors

'Division of Structural and Functional Genomics, Center for Genome Science, National Institute of Health, 363-951 Chungchengbuk-Do, Republic of Korea Full list of author information is available at the end of the article
}

Development of OA is the consequence of interaction between external stimuli, such as mechanical loading and the structure and physiology of the joint [3]. Risk factors of OA include obesity, knee injury, previous knee surgery, and occupational bending and lifting [4]. Symptoms may include joint pain, tenderness, stiffness, locking, and sometimes an effusion from the affected joint. Various causes including genetic predisposition, developmental, metabolic, and mechanical deficits can initiate inflammatory processes that lead to loss of cartilage and clinically overt OA. Not only mechanical and inflammatory stimuli to joint but also genetic susceptibility to such stimuli contribute to the pathogenesis of OA.

Recently, a number of genetic association studies for OA have been performed using candidate gene approaches or genome-wide association studies (GWASs). 
Several genes have been identified as associated with OA. GDF5 [5,6], DUS4L [7], 7q22 [8], MCF2L [9] and GNL3 [10] meet the strict criteria of genome-wide significance level with a P-value of $<5 \times 10^{-8}$. In addition to these genes, several candidate single-nucleotide polymorphisms (SNPs) in FRZB, DIO2, and SMAD2 are regarded as potentially associated genes [11-13]. However, the considerable number of SNPs in OA most likely reflects the polygenic nature of the disease with modest effect sizes for individual genes [14] and may be related to the somewhat inconclusive findings of these studies. Hence, novel approaches beyond GWASs are warrant.

Copy number variations (CNVs) account for a major proportion of human genetic polymorphisms and have been predicted to play an important role in genetic susceptibility to common diseases [15]. CNVs are one type of promising structural variation beyond SNPs, which affect only a single nucleotide. CNVs may range from $\sim 1$ kilobase to several megabases in size and can result in cellular dysfunction. CNVs associated with OA have not been elucidated.

The purpose of this study is to identify candidate CNVs associated with OA. To examine the contribution of a $\mathrm{CNV}$ region to $\mathrm{OA}$, we carried out a genome-wide association analysis between selected $\mathrm{CNV}$ regions and OA. This study revealed potential genetic variants that had not been previously reported from GWASs.

\section{Methods}

\section{Study population}

A total of 10038 individuals from the Korean Genome and Epidemiology Study, which is an ongoing prospective community-based epidemiology study in the communities of Ansung (rural) and Ansan (urban) were included. From that group, 838 individuals (371 OA cases and 467 healthy controls) who consented to X-ray examination were genotyped with NimbleGen HD2 $3 \times 720 \mathrm{~K}$ comparative genomic hybridization array $(\mathrm{aCGH})$. All the participants including controls provided written informed consent. And the study was approved by the ethics committee of Korea Centers for Disease Control and Prevention Institutional Review Board. The basic characteristics of the study participants are summarized in Table 1.

\section{Diagnostic criteria}

OA phenotype was diagnosed based on clinical and radiographic findings without assessment of symptoms.
Alternative methods of assessment such as self-reported and symptomatic OA could not be used in this study. Participants who agreed to undergo X-rays had both wrists and both knees examined. Radiographic OA was assessed based on the Kellgren/Lawrence (K/L) grading system. Patients with K/L grades of 2 or higher in the wrists or knees were diagnosed as OA [16].

\section{CNV genotyping platform}

NimbleGen $3 \times 720$ aCGH was used for CNV calling. This platform provides more than 720000 probes. A total of 360000 probes distributed uniformly across the entire human genome, whereas another 360000 probes distributed on targeted location. The median inter-probe spacing of the backbone is $<5 \mathrm{~kb}$. DNA from the NA10851 cell line was used as a reference for the aCGH to extract signal intensity ratio with hg18/NCBI build 36 . To adjust systemic biases that are present in microarray experiment, all of samples passed experimental control metrics such as chromosome $\mathrm{X}$ shift and mad.1dr with NimbleScan v.2.5. After quality control, signal intensity ratio of each probe was converted into $\log 2$ scale with the positions of probes.

\section{CNV calling}

To conduct reliable CNV association analyses, we divided the CNV calling process into two stages: CNV detection and $\mathrm{CNV}$ genotyping (Additional file 1). For $\mathrm{CNV}$ detection, the Genome Alteration Detection Analysis algorithm (GADA) was used for CNV discovery with $\mathrm{T}=10$, alpha $=0.2$, and MinSegLen $=10$ [17]. An average $\log _{2}$ ratio of \pm 0.25 of probes was set as a cut-off value to define the CNV region. To overcome the limitation of single algorithm detection, we tested CNV discovery with several parameters to find best parameter using known $\mathrm{CNV}$ region. Consequently, we select best parameter with highly concordant with known CNV region.

\section{Genotype estimation}

We used the software package, CNVtools with default parameters to assign individuals to each CNV genotype [18]. Particularly, estimated genotype from a linear discrimination function (LDF) of CNVtools was used for association analysis (Additional file 2). A mixture-based model used in CNVtools can separate samples into more exact CNV genotype group. To do this, all individuals of each $\mathrm{CNV}$ regions from the $\mathrm{CNV}$ detection with GADA

Table 1 Basic characteristics of study subjects

\begin{tabular}{|c|c|c|c|c|c|c|c|c|c|c|c|}
\hline \multirow[t]{2}{*}{ OA phenotype } & \multicolumn{2}{|c|}{ Number } & \multicolumn{3}{|c|}{ Gender (\%women) } & \multicolumn{3}{|c|}{ Age (mean) } & \multicolumn{3}{|c|}{$\mathrm{BMI}^{\mathrm{a}}$ (mean) } \\
\hline & Cases & Controls & Cases & Controls & $P$-value & Cases & Controls & $P$-value & Cases & Controls & $P$-value \\
\hline ROA One definite osteophyte (original $K \geq 2$ ) & 371 & 467 & $84 \%$ & $80 \%$ & 0.1974 & 62.22 & 61.23 & 0.023 & 25.55 & 24.28 & $1.3 \times 10^{-7}$ \\
\hline
\end{tabular}


were clustered according to the $\log _{2}$ ratio between test sample and reference sample. Additional file 3 shows type of CNV class. It is not easy to discriminant exact $\mathrm{CNV}$ genotype in the single-class CNVs, in which all individuals of the $\mathrm{CNV}$ region belonged to one cluster. On the contrary, multi-class $\mathrm{CNVs}$ that consisted of more than two clusters can assign highly confident $\mathrm{CNV}$ genotype. Therefore, only well-clustered multi-class CNV regions were included for further study. All CNV regions were also manually inspected.

Given low frequency or rare CNVs, if the number of individuals in a cluster was more than three (tripleton), we regarded this cluster as an independent cluster, whereas if the number of individuals in a cluster was two or fewer (doubleton), we did not consider this cluster as a new cluster.

\section{Statistical analysis}

Statistical analyses were performed to identify significant variant associations with hand and/or knee OA using the $\mathrm{R}$ package version 3.0.2. Logistic regression analysis adjusting for gender, age and BMI as covariates was used to calculate the statistical significance for cases-control analysis. Regarding the genotype coding scheme, 0 and 1 were used for two-class $\mathrm{CNV}$ regions. Heterozygous deletion (one copy) and normal (two copies) were coded as 0 and 1, respectively. For three-class $\mathrm{CNV}$ regions, homozygous deletion (zero copies), heterozygous deletion (one copy) and normal (two copies) were coded as 0,1 , and 2 , respectively.

\section{Validation of CNVs}

To verify estimated CNV regions, we carried out quantitative PCR using the TaqMan Copy Number Assay (Life Technologies, Foster City, USA) according to the manufacturer's protocols. In total, four pre-designed and two customized probes were used to validate the existence of the CNV. Validation samples (cases and controls) were randomly selected from each estimated genotype cluster. All validation experiments were replicated 3 times to increase the validation accuracy. The copy number in each individual was calculated with Copy Caller v2.0 using the comparative CT method according to the manufacturer's protocols.

\section{Results}

\section{Study population of each OA type}

A total of 371 patients ( 310 women, 61 men) were thus diagnosed as having OA (Table 1). The proportion of female was $84 \%$ in cases and $80 \%$ in controls, respectively. Among 371 cases, 204 and 167 were cases of hand and knee OA, respectively (Table 2 ).

\section{Selection of candidate $\mathrm{CNV}$ regions}

To estimate the reliable CNV genotype of each individual, we selected multi-class CNV regions that were well separated between clusters. Additional file 3 shows an example of a multi-class cluster that we selected. In total, 1123 multi-class $\mathrm{CNV}$ regions were selected for the association study. Among these, $463 \mathrm{CNV}$ regions were two-class regions (one and two copies) and 660 were three-class regions (zero, one and two copies) (Additional file 3). In addition, we mainly focused on copy-number deletion rather than copy-number duplication due to convenience of detection and validation.

\section{Statistical analysis}

We conducted logistic regression analyses between identified CNV regions and OA. Consequently, we selected six candidate $\mathrm{CNV}$ loci that were associated with $\mathrm{OA}$ and that ranged from about $\sim 1 \mathrm{~kb}$ to $18.5 \mathrm{~kb}$ (Table 3). Four CNV regions were within genic region (intron), and the other two regions were in non-genic region (inter-genic). After adjusting for gender, age and BMI, the P-values of the six CNV regions still satisfied a nominal P-value threshold of $<0.05$ in hand and/or knee OA.

\section{Experimental validation to assess accuracy}

To assess the accuracy of CNV estimation, we performed validation experiments with the six significant CNV regions. Table 4 shows the validation results of these six regions. The positive predictive value was applied as a measurement standard of accuracy and indicated the proportions of positive results in the $\mathrm{CNV}$ validations. The average positive predictive value of our $\mathrm{CNV}$ validation results was 0.973 , indicating that the $\mathrm{CNV}$ genotype estimation was highly reliable. Additional file 4 shows the TaqMan assay result of the CNV on chromosome 8 . The estimated CNV genotype (Additional file 4 (A)) was highly concordant with the validated genotype (Additional file 4 (B)). Except for the CNV region on chromosome 9, the genotypes of the five regions were zero, one, and two copies (Table 4).

\section{Discussion}

In this study we conducted a genome-wide association study of CNVs in OA to identify genomic regions that confer susceptibility and/or protection. The genomewide significance level following Bonferroni correction for the $1123 \mathrm{CNV}$ regions in this study was $4.5 \times 10^{-5}$. Because not every variant that has a functional impact always satisfies a genome-wide significant level and because relatively small sample size can undermine the reliability of true variants, some variants with evidence obtained from genetic study are suggested to have functional effects, even though none of identified variants meet a genome-wide significance level. Thus, we investigated 
Table 2 Number of cases and controls in each OA type

\begin{tabular}{|c|c|c|c|c|c|c|c|}
\hline \multirow[t]{2}{*}{ Study } & \multirow{2}{*}{$\begin{array}{l}\text { Classification } \\
\text { system }\end{array}$} & \multirow{2}{*}{$\begin{array}{l}\text { Cut-off } \\
\text { value for } O A\end{array}$} & \multirow[t]{2}{*}{ Exact OA definition } & \multicolumn{2}{|c|}{ Knee OA } & \multicolumn{2}{|c|}{ Wrist OA } \\
\hline & & & & Cases & Controls & Cases & Controls \\
\hline KARE $^{a}$ & K/L score & 2 & One definite osteophyte (original $K \geq 2$ ) & 167 & 467 & 204 & 467 \\
\hline
\end{tabular}

${ }^{\mathrm{a} K A R E}$ : the Korea Association Resource project.

functional relationships between $\mathrm{OA}$ and $\mathrm{CNV}$ regions that reached nominal significance $(\mathrm{P}<0.05)$. Consequently, we identified six OA genomic regions that confer susceptibility and/or protection. These regions included TNKS, CA10, POSTN, MAMDC2, KCND3, and ME3 which encompassed or were near CNV regions.

The canonical Wnt/ $\beta$-catenin signaling pathway has been implicated in the pathogenesis of osteoarthritis [19]. Increased $\beta$-catenin accumulations have been observed in degenerative cartilage, suggesting that activated Wnt signaling might contribute to progression of osteoarthritis by downstream target genes such as matrix metalloproteinases (MMPs) [20]. For instance, the secreted Wnt antagonist Dickkopf-1 is related with slowed progression of hip OA in elderly women [21] and the small molecule XAV939, which selectively inhibits $\beta$-cateninmediated transcription, is associated with protecting cartilage degradation in a rat osteoarthritis model [22]. Tankyrase, which is encoded by TNKS, bind directly to axin, a negative regulator of the canonical Wnt/ $\beta$-catenin signaling pathway, forming a destruction complex with glycogen synthase kinase $3 \beta$ (GSK-3 $\beta$ ) and adenomatous polyposis coli (APC) to degrade $\beta$-catenin [23]. Inhibition of TNKS by treating the compound XAV939 or small interfering mediated silencing of TNKS can cause an increase in levels of axin, leading to phosphorylation and degradation of $\beta$-catenin, and inhibition of target gene transcription by Wnt signaling. Thus, although further studies are needed to establish the role of TNKS in osteoarthritis through axin-mediated inhibition of Wnt signaling, it is quite possible that TNKS could be risk factor for osteoarthritis.

Figure 1 shows the chromosomal position of the copy number deletion of third intron of TNKS. Interestingly, several transcription factor binding sites as determined by ENCODE of UCSC genome browser (http://genome. ucsc.edu/ENCODE) overlapped with this CNV region (Figure 1B). We speculated that copy number deletion of this region may directly or indirectly influence the binding of transcription factors, possibly including JunD, which belongs to the Jun family of activator protein 1 transcriptional regulators. A recent study reported that JunD-deficient immortalized fibroblast exhibit increased proliferation [24]. JunD also regulates interleukin-6 and matrix metalloproteinase- 9 transcription in hepatic stellate cells, indicating that JunD may be a key profibrogenic regulator [25]. Copy number deletion of TNKS was associated with a 1.37-fold decreased risk for OA (Table 2).

CA10 gene encodes a protein that belongs to the carbonic anhydrase family of zinc metalloenzymes, which catalyze the reversible hydration of carbon dioxide [26]. The protein encoded by $C A 10$ is an acatalytic member of the alpha-carbonic anhydrase subgroup, which includes major players in many physiological processes including renal and male reproductive tract acidification and bone resorption. Carbonic anhydrases are thought to be involved in bone mineral solubilization and digestion of organic bone matrix by acid proteases. The rapid conversion of carbon dioxide to bicarbonate and protons occurs while maintaining a normal intracellular $\mathrm{pH}$ in osteoclasts [27]. The use of an inhibitor of carbonic anhydrase is associated with a bone-sparing effect, as shown by measurement of spinal bone mineral density [28]. Moreover, the intronic variants, rs2106329 in CA10 shows strong association with osteoporosis in Japanese women [29]. A homozygous A allele at rs2106329 is a protective factor for osteoporosis. In our study, copy number deletion on $C A 10$ was associated with a 1.69-fold decrease risk for hand OA as compared with a normal copy number (Table 3 ). Copy number deletion can also be a protective factor for OA.

Table 3 Summary of association result

\begin{tabular}{|c|c|c|c|c|c|c|c|c|c|}
\hline \multirow[t]{2}{*}{ Chr } & \multirow[t]{2}{*}{ Start } & \multirow[t]{2}{*}{ End } & \multirow[t]{2}{*}{ Length (bp) } & \multirow[t]{2}{*}{ Gene } & \multirow[t]{2}{*}{ OA type } & \multicolumn{2}{|c|}{ CNV genotype frequency (\%) ${ }^{a}$} & \multirow[t]{2}{*}{ OR $(95 \% \mathrm{Cl})^{\mathrm{b}}$} & \multirow{2}{*}{$\begin{array}{l}\text { Adjusted } \\
\text { P-value }^{c}\end{array}$} \\
\hline & & & & & & Cases $(0 / 1 / 2)$ & Controls $(0 / 1 / 2)$ & & \\
\hline 1 & 112490851 & 112509390 & 18539 & Upstream of KCND3 & Hand & 23.0/52.9/24.0 & $13.1 / 52.5 / 34.5$ & $0.41(0.25-0.68)$ & $5.8 \times 10^{-4}$ \\
\hline 8 & 9535412 & 9536779 & 1367 & Intron of TNKS & Hand, Knee & 2.4/28.8/68.7 & $4.3 / 34.5 / 61.2$ & $1.37(1.02-1.85)$ & $3.5 \times 10^{-2}$ \\
\hline 9 & 71952096 & 71953157 & 1061 & Intron of MAMDC2 & Knee & $-/ 7.2 / 92.8$ & $-/ 3.0 / 97.0$ & $0.36(0.16-0.86)$ & $1.9 \times 10^{-2}$ \\
\hline 11 & 85981796 & 85983048 & 1252 & Intron of ME3 & Hand & $6.9 / 33.3 / 59.8$ & $11.1 / 40.5 / 48.4$ & $1.70(1.21-2.40)$ & $2.4 \times 10^{-3}$ \\
\hline 13 & 36971042 & 36976531 & 5489 & Downstream of POSTN & Hand, Knee & 2.4/31.3/66.3 & $1.5 / 22.5 / 76.0$ & $0.64(0.47-0.87)$ & $4.9 \times 10^{-3}$ \\
\hline 17 & 47477132 & 47478158 & 1026 & Intron of CA10 & Hand & $1.5 / 19.1 / 79.4$ & 2.8/26.6/70.7 & $1.69(1.14-2.55)$ & $1.02 \times 10^{-2}$ \\
\hline
\end{tabular}

${ }^{\mathrm{a}}$ Designations for the CNV genotype are as indicated in the Methods. A dash indicates a zero-copy CNV region. OR ${ }^{\mathrm{b}}$ and Adjusted P-value ${ }^{\mathrm{c}}$ were adjusted by gender, age, and body mass index; OR: odds ratio, Cl: Confidence interval. 
Table 4 Result of TaqMan copy number assays

\begin{tabular}{llllllll}
\hline Chr & Start & End & Probe ID & CNV genotype $\mathbf{a}^{\mathbf{a}}$ & TP & FP $^{\text {PPV }}$ \\
\hline Chr1 & 112490851 & 112509390 & Hs_03201731_cn & $0,1,2$ & 179 & 9 \\
Chr8 & 9535412 & 9536779 & Hs_04345744_cn & $0,1,2$ & 73 & 1 \\
Chr9 & 71952096 & 71953157 & Hs_06899364_cn & 1,2 & 58 & 1 \\
Chr11 & 85981796 & 85983048 & Hs_03777027_cn & $0,1,2$ & 70 & 4 \\
Chr13 & 36971042 & 36976531 & CCT956B & $0,1,2$ & 85 & 3 \\
Chr17 & 47477132 & 47478158 & CCD1TXG & $0,1,2$ & 86 & 0.95 \\
\hline
\end{tabular}

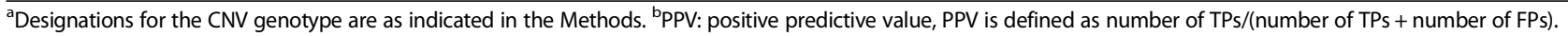

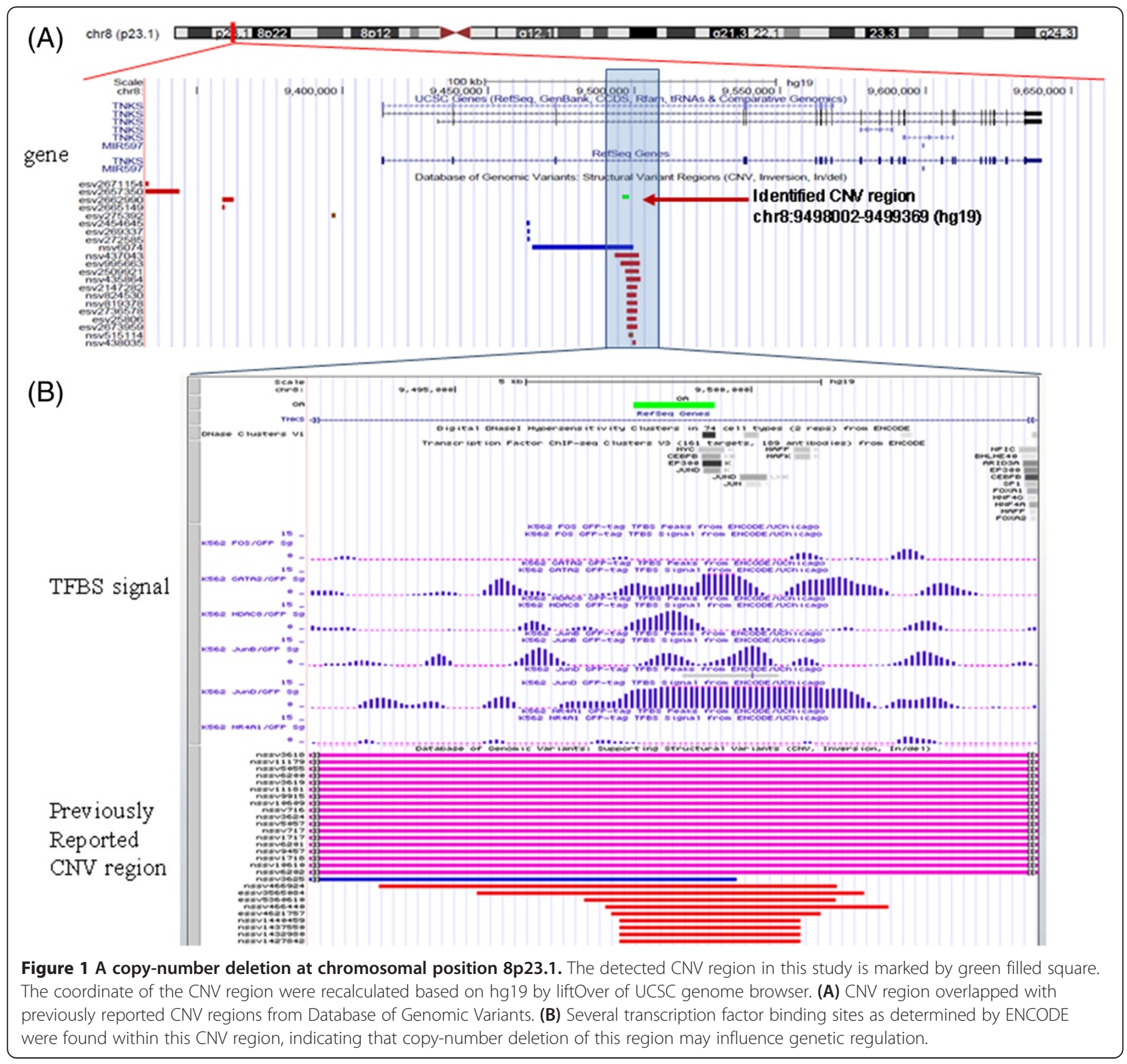


POSTN encodes periostin, an osteoblast-specific factor that functions as a ligand for $\alpha \mathrm{V} / \beta 3$ and $\alpha \mathrm{V} / \beta 5$ integrins to support adhesion and migration of epithelial cells [30]. Periostin is a matricellular glutamate-containing protein that is expressed in connective tissues including bone, periosteum, and ligament [31]. Periostin is believed to function in the regulation of bone formation and, with its preferential localization in the periosteum, may play a role in fracture repair during the early process of bone regeneration [31]. In response to mechanical stress, periostin expression is up-regulated and collagen fibrillogenesis and matrix organization occur to preserve tissue integrity and function [32]. Periostin knock-out mice show altered cortical bone microarchitecture and lower bone mineral density [33]. Periostin appears to influence the properties of bone materials and damage accumulation and repair, including local modeling/remodeling processes in response to fatigue [34]. Periostin deficiency may influence the propensity for fatigue fractures [34], and may accelerate the pathogenesis of $\mathrm{OA}$ in cooperation with an imbalance in bone repair mechanisms. Similar to previously reported study, in our study, copy number deletion downstream of POSTN was associated with an increase in the risk for hand and/or knee OA compared with the normal copy number. However, we did not find a relationship between this CNV region and POSTN.

Also, we could not find a biological role of $M A M D C 2$, $K C N D 3$, and $M E 3$ in the pathogenesis of OA.

The present study has some limitations. First, diagnosis of OA was radiologically based (i.e., using X-ray) without assessment of symptoms. Different methods of assessment such as self-reported and symptomatic OA could not be available in population-based study samples. This limitation has resulted in small sample size of discovery stage and a difficulty of collecting samples for replication analysis. Second, because of first limitation which was mentioned above, we did not success to find radiographic case samples in independent general population samples. Eventually, we did not conduct a replication study, which is highly desirable with association studies. This limitation can be solved through a meta-analysis with other data in the future. To reduce heterogeneity of meta-analysis, we provide our cohort information such as exact phenotype definition, cut-off, and number of cases of each OA type. Third, knee and hand OA were analyzed together. OA of the knee can be a component of a generalized diathesis, including OA of the hand, which may be inherited. The OA phenotype is heterogeneous and occurs at specific joint locations, in a more generalized form, or with and without clinical presentation. Each phenotype has its own heritability estimate [35]. Fourth, the sample size was relatively small, and this reduced the power of the analysis. Fifth, six novel CNVs were located not in exons but in introns or were intergenic. Despite these limitations, our study has the following strengths. First, the present study describes the first CNVs to be reported regarding OA using high-resolution chip. Second, we were able to define precise disease phenotype using X-rays of all the participants.

\section{Conclusions}

We identified several previously unreported $\mathrm{CNV}$ loci that predisposed to or provided protection from OA. Identification of the associated variants at these genomic loci and their potential functional consequences will lead to better understanding of the pathogenesis of OA. Although this study has some limitations, further functional investigations of these genes are warranted to fully characterize the association.

\section{Additional files}

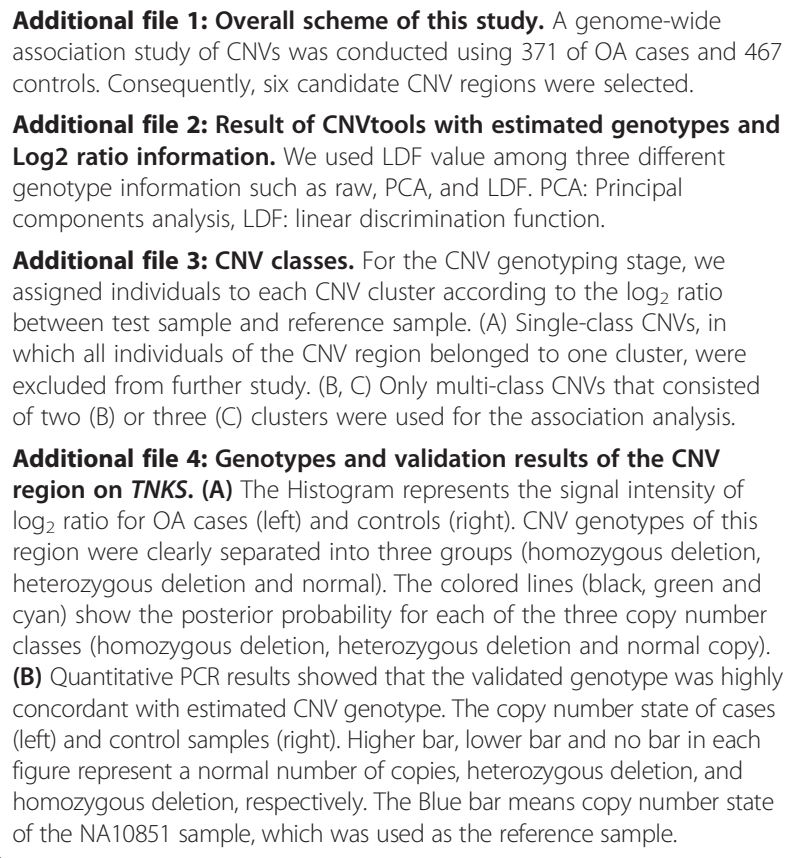

Additional file 3: CNV classes. For the CNV genotyping stage, we assigned individuals to each CNV cluster according to the $\log _{2}$ ratio between test sample and reference sample. (A) Single-class CNVs, in which all individuals of the CNV region belonged to one cluster, were excluded from further study. (B, C) Only multi-class CNVs that consisted of two (B) or three $(C)$ clusters were used for the association analysis.

Additional file 4: Genotypes and validation results of the CNV region on TNKS. (A) The Histogram represents the signal intensity of $\log _{2}$ ratio for OA cases (left) and controls (right). CNV genotypes of this region were clearly separated into three groups (homozygous deletion, heterozygous deletion and normal). The colored lines (black, green and cyan) show the posterior probability for each of the three copy number classes (homozygous deletion, heterozygous deletion and normal copy). (B) Quantitative PCR results showed that the validated genotype was highly concordant with estimated CNV genotype. The copy number state of cases (left) and control samples (right). Higher bar, lower bar and no bar in each figure represent a normal number of copies, heterozygous deletion, and homozygous deletion, respectively. The Blue bar means copy number state of the NA10851 sample, which was used as the reference sample.

\section{Abbreviations}

OA: Osteoarthritis; GWAS: Genome-wide association study; SNP: Single nucleotide polymorphism; CNV: Copy number variation; aCGH: Comparative genomic hybridization array; BMl: Body mass index.

\section{Competing interests}

The authors declare that they have no competing interests.

\section{Authors' contributions}

SM: manuscript writing, conception and design, data analysis. BK: manuscript writing, analysis and interpretation of data. MYH: data analysis, data management. YL: data analysis. SP: data analysis. JHO: validation experiment. YJK: validation experiment. HSL: manuscript writing of a part of discussion. NHK: data manual curation, data analysis. YJK: data manual curation, data analysis. DHK: data collection, result interpretation. BGH: comments for project design, final approval of the version to be published. BJK: conception and design, project management. JL: data collection, conception and design, project management. All authors have read and approved the final manuscript for publication. 


\section{Acknowledgements}

This work was supported by intramural grants from the Korea National Institute of Health (2012-N73004-00 \& 2013-NG73002-00) and also provided with biospecimens data from the Korea Genome Analysis Project (4845-301), the Korean Genome and Epidemiology Study (4851-302), and Korea Biobank Project (4851-307).

\section{Author details}

'Division of Structural and Functional Genomics, Center for Genome Science, National Institute of Health, 363-951 Chungchengbuk-Do, Republic of Korea. ${ }^{2}$ Department of Internal Medicine, Seoul National University Hospital, 110-744 Seoul, Republic of Korea. ${ }^{3}$ Department of Social and Preventive Medicine, Hallym University College of Medicine, 200-702 Chunchun, Republic of Korea. ${ }^{4}$ Department of Biostatistics, Soonchunhyang University, College of Medicine, 140-743 Seoul, Republic of Korea.

\section{Received: 27 June 2014 Accepted: 17 March 2015}

\section{Published online: 04 April 2015}

\section{References}

1. Brandt KD, Dieppe P, Radin EL. Etiopathogenesis of osteoarthritis. Rheum Dis Clin North Am. 2008;34(3):531-59.

2. Guccione AA, Felson DT, Anderson JJ, Anthony JM, Zhang Y, Wilson PW, et al. The effects of specific medical conditions on the functional limitations of elders in the Framingham Study. Am J Public Health. 1994;84(3):351-8.

3. van der Kraan PM. Understanding developmental mechanisms in the context of osteoarthritis. Curr Rheumatol Rep. 2013;15(6):333.

4. Felson DT, Lawrence RC, Dieppe PA, Hirsch R, Helmick CG, Jordan JM, et al. Osteoarthritis: new insights. Part 1: the disease and its risk factors. Ann Intern Med. 2000;133(8):635-46.

5. Miyamoto $Y$, Mabuchi A, Shi D, Kubo T, Takatori Y, Saito S, et al. A functional polymorphism in the $5^{\prime}$ UTR of GDF5 is associated with susceptibility to osteoarthritis. Nat Genet. 2007:39(4):529-33.

6. Valdes AM, Evangelou E, Kerkhof HJ, Tamm A, Doherty SA, Kisand K, et al. The GDF5 rs143383 polymorphism is associated with osteoarthritis of the knee with genome-wide statistical significance. Ann Rheum Dis. 2011;70(5):873-5.

7. Evangelou E, Valdes AM, Kerkhof HJ, Styrkarsdottir U, Zhu Y, Meulenbelt I, et al. Meta-analysis of genome-wide association studies confirms a susceptibility locus for knee osteoarthritis on chromosome 7q22. Ann Rheum Dis. 2011;70(2):349-55.

8. Kerkhof HJ, Lories RJ, Meulenbelt I, Jonsdottir I, Valdes AM, Arp P, et al. A genome-wide association study identifies an osteoarthritis susceptibility locus on chromosome 7q22. Arthritis Rheum. 2010;62(2):499-510.

9. Day-Williams AG, Southam L, Panoutsopoulou K, Rayner NW, Esko T, Estrada $\mathrm{K}$, et al. A variant in MCF2L is associated with osteoarthritis. Am J Hum Genet. 2011;89(3):446-50.

10. Davies RW, Wells GA, Stewart AF, Erdmann J, Shah SH, Ferguson JF, et al. A genome-wide association study for coronary artery disease identifies a novel susceptibility locus in the major histocompatibility complex. Circ Cardiovasc Genet. 2012;5(2):217-25.

11. Hochberg MC, Yerges-Armstrong L, Yau M, Mitchell BD. Genetic epidemiology of osteoarthritis: recent developments and future directions. Curr Opin Rheumatol. 2013;25(2):192-7.

12. Meulenbelt I. Osteoarthritis year 2011 in review: genetics. Osteoarthritis Res Soc. 2012;20(3):218-22

13. van Meurs JB, Uitterlinden AG. Osteoarthritis year 2012 in review: genetics and genomics. Osteoarthritis Res Soc. 2012;20(12):1470-6.

14. Panoutsopoulou K, Southam L, Elliott KS, Wrayner N, Zhai G, Beazley C, et al. Insights into the genetic architecture of osteoarthritis from stage 1 of the arcOGEN study. Ann Rheum Dis. 2011;70(5):864-7.

15. Wellcome Trust Case Control C, Craddock N, Hurles ME, Cardin N, Pearson RD, Plagnol V, et al. Genome-wide association study of CNVs in 16,000 cases of eight common diseases and 3,000 shared controls. Nature. 2010;464(7289):713-20

16. Kellgren JH, Lawrence JS. Radiological assessment of osteo-arthrosis. Ann Rheum Dis. 1957;16(4):494-502.

17. Pique-Regi R, Monso-Varona J, Ortega A, Seeger RC, Triche TJ, Asgharzadeh S. Sparse representation and Bayesian detection of genome copy number alterations from microarray data. Bioinformatics. 2008;24(3):309-18.

18. Barnes C, Plagnol V, Fitzgerald T, Redon R, Marchini J, Clayton D, et al. A robust statistical method for case-control association testing with copy number variation. Nat Genet. 2008;40(10):1245-52.
19. Corr M. Wnt-beta-catenin signaling in the pathogenesis of osteoarthritis. Nat Clin Pract Rheumatol. 2008:4(10):550-6.

20. Monfort J, Garcia-Giralt N, Lopez-Armada MJ, Monllau JC, Bonilla A, Benito P, et al. Decreased metalloproteinase production as a response to mechanical pressure in human cartilage: a mechanism for homeostatic regulation. Arthritis Res Ther. 2006;8(5):R149.

21. Lane NE, Nevitt MC, Lui LY, de Leon P, Corr M, Study of Osteoporotic Fractures Research G. Wnt signaling antagonists are potential prognostic biomarkers for the progression of radiographic hip osteoarthritis in elderly Caucasian women. Arthritis Rheum. 2007:56(10):3319-25.

22. Zeng $L$, Wang $W$, Rong XF, Zhong $Y$, Jia $P$, Zhou GQ, et al. Chondroprotective effects and multi-target mechanisms of Icariin in IL-1 beta-induced human SW 1353 chondrosarcoma cells and a rat osteoarthritis model. Int Immunopharmacol. 2014;18(1):175-81.

23. Huang SM, Mishina YM, Liu S, Cheung A, Stegmeier F, Michaud GA, et al. Tankyrase inhibition stabilizes axin and antagonizes Wnt signalling. Nature. 2009:461(7264):614-20.

24. Zenz R, Eferl R, Scheinecker C, Redlich K, Smolen J, Schonthaler HB, et al. Activator protein 1 (Fos/Jun) functions in inflammatory bone and skin disease. Arthritis Res Ther. 2008;10(1):201.

25. Smart DE, Green K, Oakley F, Weitzman JB, Yaniv M, Reynolds G, et al. JunD is a profibrogenic transcription factor regulated by Jun N-terminal kinaseindependent phosphorylation. Hepatology. 2006;44(6):1432-40.

26. Pastorekova S, Parkkila S, Pastorek J, Supuran CT. Carbonic anhydrases: current state of the art, therapeutic applications and future prospects. J Enzyme Inhib Med Chem. 2004;19(3):199-229.

27. Rousselle AV, Heymann D. Osteoclastic acidification pathways during bone resorption. Bone. 2002;30(4):533-40.

28. Pierce Jr WM, Nardin GF, Fuqua MF, Sabah-Maren E, Stern SH. Effect of chronic carbonic anhydrase inhibitor therapy on bone mineral density in white women. J Bone Min Res. 1991;6(4):347-54

29. Mori S, Kou I, Sato H, Emi M, Ito H, Hosoi T, et al. Nucleotide variations in genes encoding carbonic anhydrase 8 and 10 associated with femoral bone mineral density in Japanese female with osteoporosis. J Bone Miner Metab. 2009;27(2):213-6.

30. Gillan L, Matei D, Fishman DA, Gerbin CS, Karlan BY, Chang DD. Periostin secreted by epithelial ovarian carcinoma is a ligand for alpha(V)beta(3) and alpha(V)beta(5) integrins and promotes cell motility. Cancer Res. 2002;62(18):5358-64.

31. Merle B, Garnero P. The multiple facets of periostin in bone metabolism. Osteoporosis Int. 2012:23(4):1199-212.

32. Rani S, Barbe MF, Barr AE, Litvin J. Induction of periostin-like factor and periostin in forearm muscle, tendon, and nerve in an animal model of work-related musculoskeletal disorder. J Histochem Cytochem. 2009;57(11):1061-73.

33. Bonnet N, Standley KN, Bianchi EN, Stadelmann V, Foti M, Conway SJ, et al. The matricellular protein periostin is required for sost inhibition and the anabolic response to mechanical loading and physical activity. J Biol Chem. 2009;284(51):35939-50

34. Bonnet N, Gineyts E, Ammann P, Conway SJ, Garnero P, Ferrari S. Periostin deficiency increases bone damage and impairs injury response to fatigue loading in adult mice. PLoS One. 2013;8(10):e78347.

35. van den Berg WB. Osteoarthritis year 2010 in review: pathomechanisms. Osteoarthritis Res Soc. 2011;19(4):338-41.

\section{Submit your next manuscript to BioMed Central and take full advantage of:}

- Convenient online submission

- Thorough peer review

- No space constraints or color figure charges

- Immediate publication on acceptance

- Inclusion in PubMed, CAS, Scopus and Google Scholar

- Research which is freely available for redistribution 